\title{
Obliteración del seno frontal mediante injerto de tibia y plasma rico en plaquetas para el tratamiento de la osteomielitis crónica
}

\author{
M. Acosta-Feria; P. Infante-Cossío; J.M. Hernández-Guisado; E. Torres-Carranza; A. García-Perla; R. Belmonte- \\ Caro y J.L. Gutiérrez-Pérez
}

Servicio de Cirugía Maxilofacial. Hospitales Universitarios Virgen del Rocío. Sevilla.

\section{Resumen}

Objetivos. La técnica quirúrgica más eficaz en el tratamiento de la sinusitis frontal crónica complicada con una osteomielitis crónica, incluye la limpieza y legrado del seno, y la obliteración de los espacios muertos y sellado del ostium del seno frontal. Existen varios materiales autólogos y aloplásticos usados para la obliteración sinusal.

Material y métodos. Paciente intervenido de un plasmocitoma localizado en el seno frontal, que desarrolló una sinusitis frontal complicada con una osteomielitis crónica con fistulización y supuración cutánea. Se trató mediante un legrado de la cavidad del seno frontal y obliteración con injerto esponjoso de tibia y plasma rico en plaquetas (PRP).

Conclusiones. La obliteración tras limpieza y legrado del seno frontal es un tratamiento efectivo para la osteomielitis crónica sinusal. Tras la intervención quirúrgica, se comprobó la desaparición de los síntomas de nuestro paciente. No hubo recidiva de la enfermedad ni complicaciones postoperatorias. EI uso de injerto óseo de tibia y PRP puede ser una alternativa eficaz a los materiales existentes para dicha obliteración.

PALABRAS CLAVE: Seno frontal. Obliteración. Osteomielitis crónica. Tibia. Injerto óseo. Plasma rico en plaquetas.

Frontal sinus obliteration using tibial bone graft and platelet-rich plasma for the treatment of chronic osteomyelitis

Summary

Objetives. The more successfully surgical treatment for the treatment of chronic frontal sinusitis complicated with chronic osteomielitis, is the cleaning and curettage of the sinus with obliteration of the sinus

Recibido; 07-07-05. Aceptado: 18-10-05 cavity and nasofrontal duct. Several autologous and alloplastics materials are used for this obliteration.

Material and methods. We report the case of a patient previously operated on because of frontal sinus plasmocytoma who developed frontal sinusitis with chronic osteomielitis and cutaneous suppuration. He received surgical treatment with frontal sinus curettage and obliteration with tibial bone graft and platelet-rich plasma (PRP). Symptoms disappeared and there were no complications or recurrence.

Conclusions. Obliteration of the frontal sinus with tibial bone graft and PRP after sinus cleaning and curettage can be a good alternative in the treatment of chronic frontal sinus osteomielitis.

KEY WORDS: Frontal Sinus. Obliteration. Chronic Osteomyelitis. Tibia. Bone graft. Platelet-rich plasma.

Introducción

Las sinusitis crónicas de los senos paranasales pueden dar lugar a complicaciones intracraneales y extracraneales, de forma aislada o combinada. Entre las complicaciones intracraneales destacan la meningitis, la trombosis de los senos venosos, el absceso cerebral, el absceso epidural y el empiema subdural. Entre las extracraneales, pueden aparecer mucoceles, osteomielitis y tumor de Pott, además de complicaciones orbitarias 5 . Las osteomielitis crónicas se suelen manifestar con síntomas tales como dolor de cabeza, fiebre, hinchazón en la región frontal, fistulización y supuración cutánea ocasional, aunque a veces la clínica es silente. Existen una serie de factores predisponentes para su desarrollo tales como las inmunodeficiencias, la cirugía previa del seno, el consumo de cocaína, los traumatismos sobre esta zona, los trastornos mucociliares o la rinitis vasomotora. En los pacientes con una infección ya conocida del seno frontal y en los que concurran algunos de estos factores predisponentes, hay que tener un alto grado de sospecha clínica frente a la posible aparición de complicaciones y disponer de un estudio de tomografía axial computarizada (TAC), ya que el diagnóstico tardío y el 


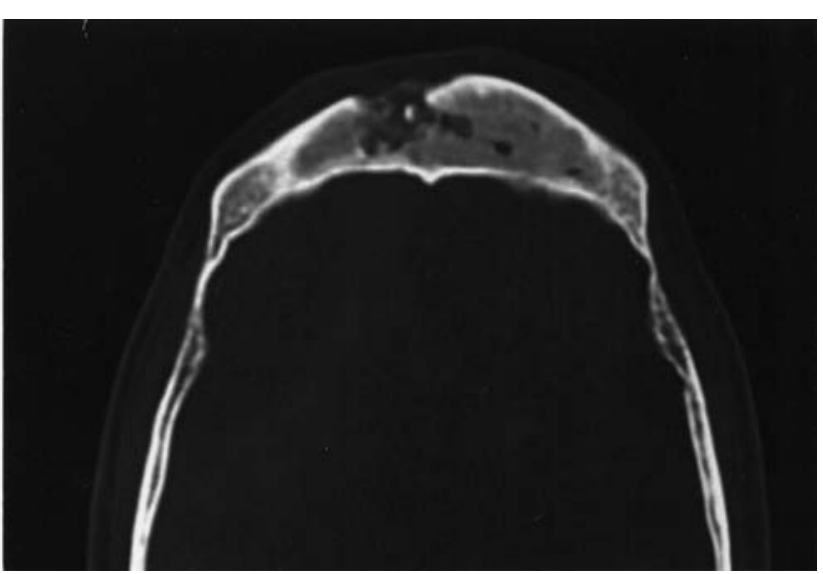

Figura 1 A. TC (axial) en el que se observa un engrosamiento del hueso frontal y ocupación de sus cavidades con un área lítica sugerente de osteomielitis con áreas de secuestro a nivel frontal.

retraso en el tratamiento pueden incrementar las tasas de morbilidad y mortalidad ${ }^{7,13,14,24}$.

El manejo terapéutico, tanto de las sinusitis frontales crónicas como de las osteomielitis crónicas, requiere no sólo un tratamiento farmacológico (antibioterapia), sino además quirúrgico ${ }^{12}$. La técnica quirúrgica que se ha demostrado más eficaz en el tratamiento de la osteomielitis frontal crónica es la realización de un colgajo osteoplástico, limpieza y legrado, y la obliteración del seno frontal. La obliteración del seno frontal se puede realizar con grasa autóloga, con colgajo de galea-pericráneo o con diversos biomateriales ${ }^{19,20}$. Aunque existen distintos tejidos autólogos y materiales aloplásticos usados con distintos grados de éxito, la implantación de injerto de grasa autóloga para la obliteración de la cavidad, es el método más utilizado $^{1,18}$. Otros materiales descritos en la literatura son hueso, músculo, fascia temporal, cristal bioactivo, fibras de tetrafluoroetileno, sulfato cálcico, celulosa, hidroxiapatita y cartílago liofilizado ${ }^{1,6}$.

Presentamos el caso de un paciente tratado de un plasmocitoma localizado en el seno frontal, que desarrolló una osteomielitis crónica con fistulización y supuración cutánea. Se trató mediante un legrado de la cavidad del seno frontal y obliteración con un injerto esponjoso de tibia y plasma rico en plaquetas (PRP).

\section{Caso clínico}

Paciente varón de 54 años, fumador y bebedor moderado, alérgico a penicilinas, con antecedentes de episodios de sinusitis maxilar y pansinusitis. Fue diagnosticado de un plasmocitoma del seno frontal izquierdo tras una biopsia por punción sinusal en otro centro hospitalario, donde se realizó una exéresis quirúrgica del mismo mediante una

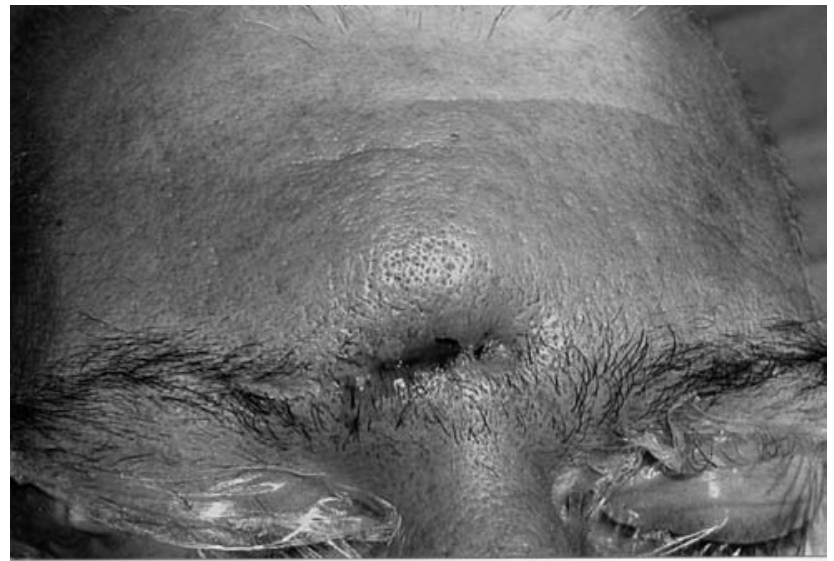

Figura 1 B. Imagen de la fistula cutánea provocada por la osteomielitis crónica del seno frontal.

incisión a nivel de ceja izquierda y una osteotomía de pared anterior del seno. Se obtuvo un fragmento de la cavidad sinusal para análisis anatomopatológico confirmando la presencia del plasmocitoma. Se realizó además un análisis histopatológico de la mucosa sinusal extirpada, que fue informada como posible mucocele abscesificado (mucopiocele).

Con posterioridad fue derivado al Servicio de Hematología de nuestro Hospital para tratamiento complementario. En la gammagrafía ósea, se observó depósito patológico del trazador en seno maxilar, huesos propios nasales y senos frontales, sospechando la presencia de mieloma. Se administraron tres ciclos de quimioterapia separados por un mes entre ellos, consistentes en vincristina, adriomicina y dexametasona + Aredia ( $1^{\mathrm{er}}$ ciclo); ciclofosfamida, etopóxido, dexametasona + Aredia $\left(2^{\circ}\right.$ ciclo); y ciclofosfamida, adriomicina, dexametasona + Aredia ( $3^{\circ}$ ciclo). A los dos días de finalizar el tercer ciclo del tratamiento quimioterápico, se observó un aumento de tumoración en región frontal, con signos de inflamación local de 24 horas de evolución. A la exploración se palpaba una tumoración blanda a nivel frontal que drenaba abundante material purulento a través de dos orificios situados en la ceja, con cefalea y fiebre de $38^{\circ} \mathrm{C}$. Se tomaron unas muestras para cultivo y antibiograma (no se aislaron microorganismos). En la $\mathrm{Rx}$ de cráneo se visualizó una zona radiolúcida a nivel del hueso frontal. Por TC y RM se confirmó la presencia de un engrosamiento del hueso frontal y ocupación de sus cavidades con un área lítica en el lado izquierdo sugerente de osteomielitis con áreas de secuestro a nivel frontal. (figura 1).

Se remitió a nuestro Servicio de Cirugía Maxilofacial y tras valoración del paciente, se decidió llevar a cabo tratamiento quirúrgico. Se realizó una incisión en alas de mariposa en la región glabelar incluyendo el trayecto fistuloso. Tras despegar el colgajo se visualizó el seno frontal que 


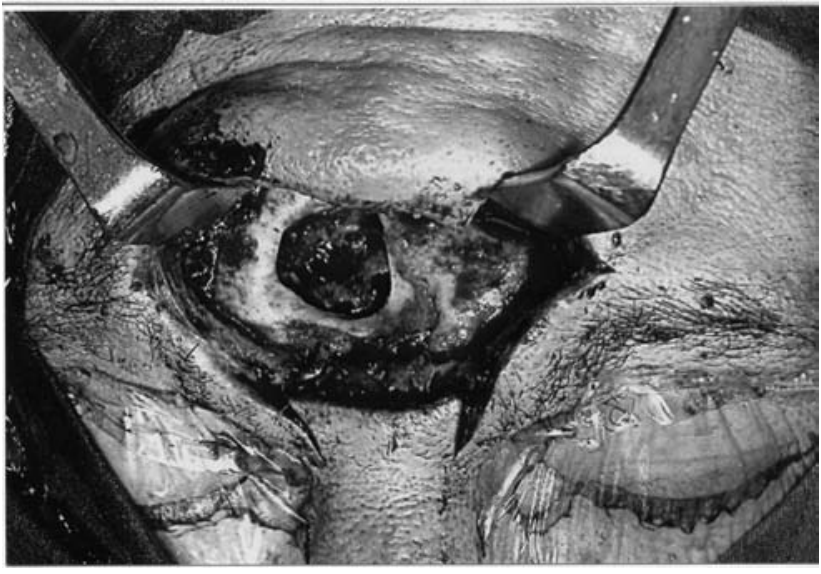

Figura 2 A. Imagen de la cavidad sinusal tras la limpieza y legrado de la misma.

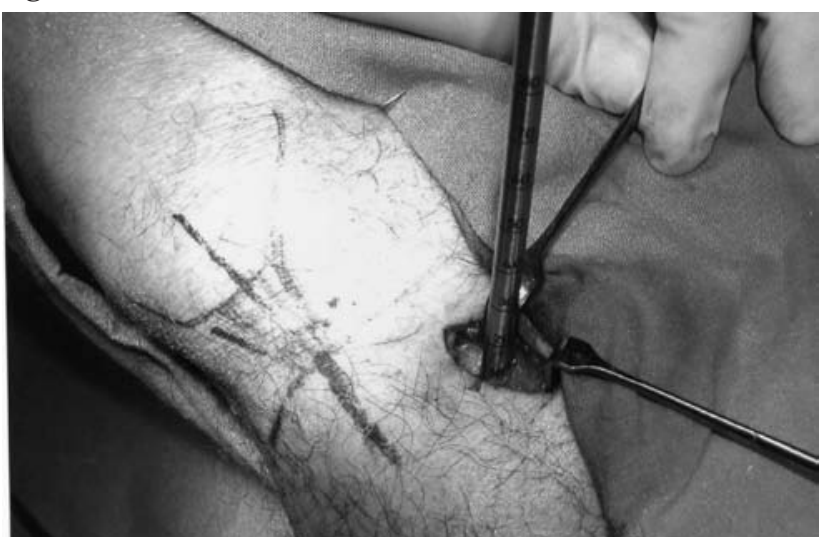

Figura 2 B. Imagen de la toma del injerto corticoesponjoso de tibia proximal derecha.

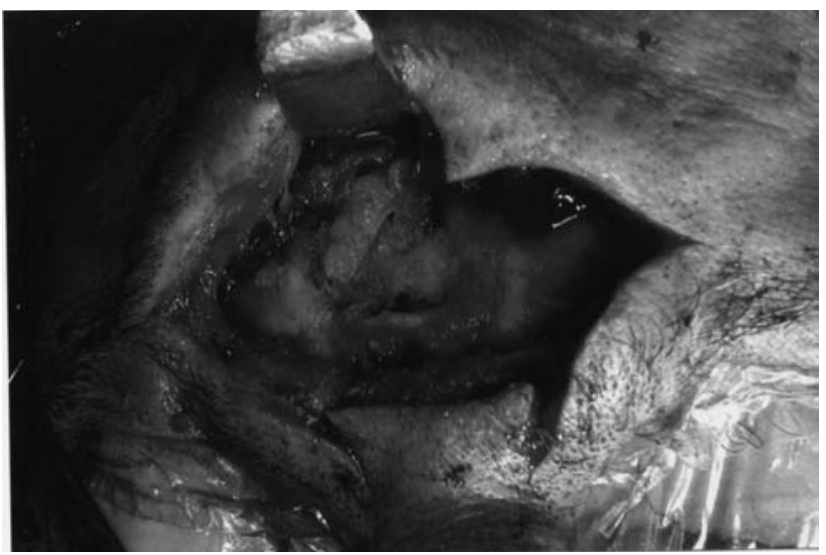

Figura 2C. Imagen de la cavidad sinusal obliterada por la mezcla del injerto corticoesponjoso de tibia más plasma rico en plaquetas $(P R P)$.

había perdido la tabla externa, se procedió a la limpieza y legrado y a fresar las paredes del seno con material rotatorio. Se tomó un injerto de $50 \mathrm{cc}$. de hueso corticoesponjoso de la tibia proximal derecha mediante un trócar, que se

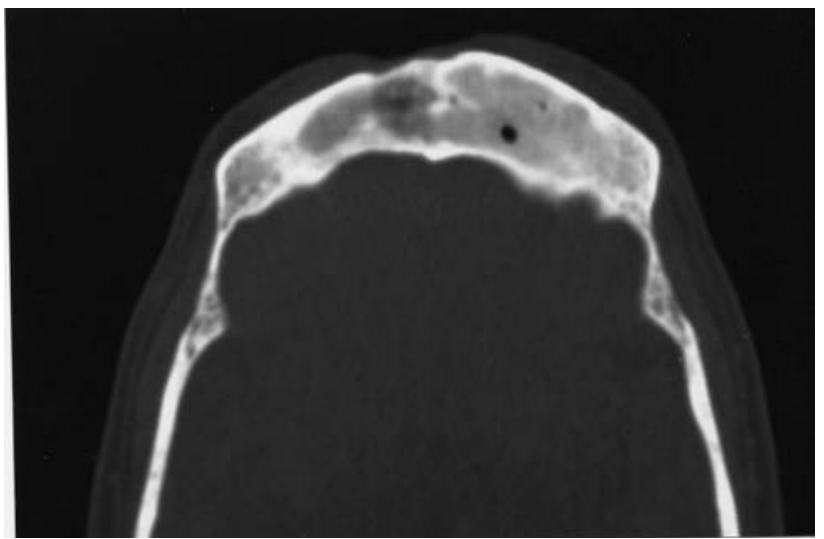

Figura 3 A. TC (axial) de control a los doce meses en los que se visualiza un relleno total de la cavidad sinusal, sin signos de enfermedad.

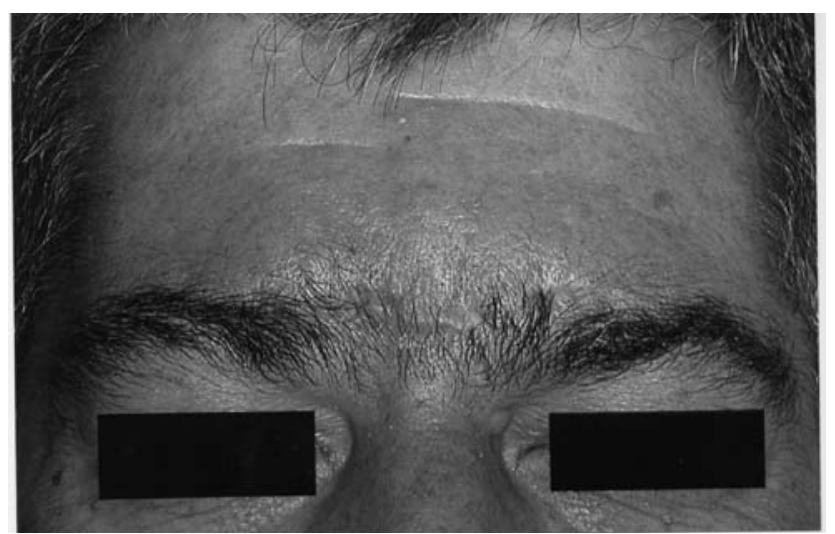

Figura 3 B. Aspecto y resultado estético del paciente al año de la intervención.

mezcló con un concentrado de fracción rica de factores de crecimiento de PRP que se había preparado previamente. Se extrajeron $250 \mathrm{cc}$ de sangre venosa para preparar el PRP, que fueron centrifugados mediante una técnica de dos fases (Platelet Concentrate Collection System PCCS; 3i/Implant

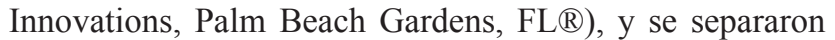
las dos fracciones. Antes de su aplicación, se activó el coágulo de PRP mediante cloruro cálcico. En total se obtuvo una mezcla de $40 \mathrm{cc}$ de material, con la que se rellenaron ambas cavidades sinusales en su totalidad. (fig. 2) Se suturó la herida quirúrgica colocándose plasma pobre en factores de crecimiento sobre la cicatriz.

Se remitió el material legrado de ambas cavidades sinusales para análisis anatomopatológico. El resultado informó de la presencia de tejido inflamatorio de tipo mixto con afectación de hueso y partes blandas, descartándose recidiva de plasmocitoma, siendo el diagnóstico de osteomielitis crónica del seno frontal.

El paciente fue dado de alta tras cinco días de hospitalización sin presentar sintomatología ni complicaciones postoperatorias. A nivel de la zona donante no se produ- 
jeron complicaciones, deambulando el paciente al alta sin molestias. Se hicieron controles de TC a los seis y doce meses de la intervención en los que se observó un relleno total de la cavidad sinusal, sin signos de enfermedad. El paciente no ha vuelto a presentar tumefacción o supuración a nivel frontal, cefaleas o fiebre. El aspecto estético de la herida quirúrgica es muy satisfactorio. (fig. 3).

\section{Discusión}

El desarrollo de una osteomielitis del seno frontal como complicación de una sinusitis frontal es poco frecuente, pero siempre hay que sospecharlo ante un paciente diagnosticado previamente de una sinusitis frontal o una pansinusitis y que refiera síntomas tales como cefalea frontal, fiebre (los dos síntomas más comunes de presentación) o supuración $^{5,12}$. El diagnóstico se basará tanto en la clínica como en las pruebas de imagen; la fundamental será una TAC, con o sin contraste. Se ha visto que existen ciertos factores que predisponen a la complicación de la sinusitis; en nuestro paciente, existían algunos muy claros, la cirugía previa sobre la región frontal y la inmunodepresión motivada por su enfermedad de base y el régimen quimioterápico.

La osteomielitis crónica de seno frontal requiere el tratamiento quirúrgico en muchas ocasiones para la curación del enfermo. La técnica de elección es el abordaje abierto del seno frontal que permita la limpieza y el legrado bajo visión directa con su obliteración. Se puede realizar, bien a través de un colgajo osteoplástico tras incisión bicoronal, con una incisión directa sobre la zona, o bien a través de una craneotomía bifrontal ${ }^{2,3,4,16,21}$. En nuestro caso llevamos a cabo un abordaje directo aprovechando la cicatriz anterior, extirpando a la vez los trayectos fistulosos cutáneos y facilitando el acceso directo al seno frontal.

Entre las complicaciones que se pueden presentar durante o tras la cirugía tenemos: deformidad frontal en forma de depresión o prominencia, edema palpebral y/o diplopia; en personas delgadas imposibilidad de extraer grasa abdominal; persistencia de la patología o recidiva por extirpación incompleta de la mucosa, obliteración incompleta, infección de tejidos injertados y ducto frontonasal abierto ${ }^{2}$.

Se han de tener en cuenta unos principios esenciales para el éxito de la obliteración sinusal ${ }^{8,16,21,25}$ :

- Extirpación meticulosa de toda la mucosa sinusal y de la cortical de las paredes del seno inmediatamente por debajo de la mucosa, preferentemente con magnificación.

- Oclusión permanente del conducto nasofrontal para formar una barrera fibrosa entre la cavidad sinusal obliterada y la cavidad nasal, previniendo el ascenso de la posible infección desde la fosa nasal y el crecimiento de la mucosa hacia el seno, con la potencial neocavitación sinusal y formación de quiste.

La obliteración sinusal con tejidos autólogos presenta una baja tasa de fracasos, siendo bien tolerados por el seno frontal. El fallo de estas técnicas, se debe a la reabsorción del tejido, infección o rechazo del injerto ${ }^{19,22}$. Para examinar el grado de obliteración del seno tras la intervención, e identificar los pacientes de riesgo de recurrencia de la enfermedad sinusal después del proceso quirúrgico, se aconseja realizar TC o $\mathrm{RM}^{18,25}$.

La obliteración del seno frontal mediante la implantación de tejido adiposo autógeno, introducido por Bergara e Itoiz, y posteriormente popularizado por Montgomery, es el método más utilizado por la mayoría de los cirujanos ${ }^{21,25}$. Complicaciones encontradas con su uso son: infección del injerto, necrosis de la grasa, infección sinusal crónica recurrente, complicaciones a nivel abdominal (abscesos, seromas o hematomas) $)^{6,17,18}$. El uso de músculo autógeno o fascia lata para la obliteración del seno frontal, esta menos extendido, aunque en algunas series se han obtenido buenos resultados ${ }^{21,25}$. El colgajo de galea pericráneo puede ser usado localmente para cubrir u obliterar defectos del cráneo y de la cara. Es un colgajo con suficiente volumen para poder obliterar el seno frontal ${ }^{17}$.

El hueso autógeno para la obliteración del seno frontal, ha sido usado en las últimas décadas. Los chips de huesos cortical rápidamente se vascularizan y se implantan para la restauración de defectos del contorno facial. Grahne, en una pequeña serie de pacientes con sinusitis crónica frontal, tratados mediante la obliteración del seno con hueso cortical autógeno obtenido de cresta ilíaca, no encontró complicaciones en los 5 años del periodo del seguimiento. Los 11 pacientes mostraron evidencia radiográfica postoperatoria de una osificación completa del seno frontal ${ }^{21}$. En la literatura revisada, no existe ningún trabajo que reporte el empleo del injerto de tibia junto con PRP en la cirugía del seno frontal. Nosotros obtuvimos el tejido óseo corticoesponjoso de la tibia y lo mezclamos con PRP, obteniendo resultado óptimo.

El injerto del hueso proximal de tibia es un procedimiento relativamente simple de realizar, con una baja tasa de complicaciones ${ }^{10}$. Se pueden obtener entre 5 y $30 \mathrm{cc} \mathrm{de}$ hueso esponjoso en partículas, tomados con cucharillas o trefinas de la meseta tibial. Si el injerto se compacta cuidadosamente en la cavidad aumenta la densidad celular, y por ende la cantidad de hueso neoformado y su duración. Se ha utilizado en cirugía craneofacial y maxilofacial en situaciones clínicas tan variadas como cirugía dento-alveolar, oncológica, malformativa, traumatológica, etc. Las complicaciones que pueden aparecer en la zona donante son la equimosis (más frecuente), molestias al deambular, seromas y rara vez parestesia, tromboflebitis o fractura tibial $^{9,15}$.

Una de las características de los injertos en partículas es 
que se pueden mezclar fácilmente con plasma rico en factores de crecimiento. Parece ser que la adición de plasma rico en factores de crecimiento acelera la cicatrización de los tejidos blandos y favorece la regeneración ósea y la angiogénesis y proliferación de células indiferenciadas en combinación con injertos de hueso autógeno. Marx y cols. en 1998, publicaron un estudio de 88 pacientes en los que, después de extirpar tumores tanto malignos como benignos, procedieron a la reconstrucción (con injertos óseos, asociados a plasma rico en plaquetas) de defectos en la continuidad mandibular, fisuras alveolares y cirugías de seno maxilar. Este estudio concluyó que los injertos en los que se había colocado plasma rico en plaquetas tenían un mayor índice de maduración del injerto, mediante análisis radiológicos, de anticuerpos monoclonales e histomorfométricos. Los factores de crecimiento obtenidos de plasma rico en plaquetas introducidos al mismo tiempo que un injerto de hueso autólogo constituyen un procedimiento útil y asequible para promover la tasa de hueso neoformado, que elimina la posibilidad de transmisión de enfermedades y reacciones inmunológicas asociadas a los preparados alogénicos $^{11,23}$.

\section{Conclusión}

El tratamiento de la osteomielitis crónica del seno frontal precisa en muchas ocasiones del tratamiento quirúrgico, para proceder a su limpieza y legrado. La obliteración del seno con diversos materiales autólogos o alogénicos es esencial para evitar recidivas. El cirujano debe conocer las propiedades biológicas y características fundamentales de los materiales de injerto autólogos, las diferentes técnicas de obtención y sus aplicaciones clínicas como materiales de relleno de cavidades óseas. Entre éstos, el injerto esponjoso de tibia y PRP para obliterar el seno frontal puede ser una alternativa eficaz.

\section{Bibliografía}

1. Acosta-Feria, M., García-Perla, A., Infante-Cossío, P., Belmonte-Caro, R., Gutiérrez-Pérez, J. L.: Intracranial abscess secondary to chronic frontal sinusitis. Rev Neurol. 2004; 39: 691-693.

2. Bertrán, J.M., Pérez, C., Martínez, A.: Colgajo osteoplástico del seno frontal. Estudio de 47 casos. Acta Otorrinolaring Esp. 1998; 48: 380-384.

3. Brook, I.: Acute and chronic frontal sinusitis. Curr Opin Pulm Med. 2003; 9: 171-174.

4. Correa, A.J., Duncavage, J.A., Fortune, D.S., Reinisch.: Osteoplastic flap for obliteration of frontal sinus Five years' experience. Otolaryngol Head Neck Surg. 1999; 121: 731735.

5. Dolan, R.W., Chowdhury, K.: Diagnosis and treatment of intracraneal complications of paranasal sinus infection. J Oral Maxillofac Surg. 1995; 53: 1080-1087.

6. Fattabi, T., Johnson, C., Steinberg, B.: Comparison of 2 preferred methods used for frontal sinus obliteration. J Oral Maxillofac Surg 2005; 63. 455-465.

7. Goldberg, A.N., Oroszlan, G., Anderson, T.D.: Complications of frontal sinusitis and their management. Otolaryngol Clin North Am. 2001; 34: 211- 225.

8. Hardy, J.M., Montgomery, W.W.: Osteoplastic frontal sinusotomy: an analysis of 250 operations. Ann. Otol. Rhinol. Laryngol 1976; 85: 523-532.

9. Hernández-Alfaro, F., Marti, C., Biosca, M.J., Gimeno, J.: Minimally invasive tibial bone harvesting under intravenous sedation. J Oral Maxillofac Surg. 2005; 63: 464-470.

10. Jaske, N., Seibert, F.J., Lorenzoni, M., Eskici, A., Pertl, C.: A modified technique of harvesting tibial cancellous bone and its use in sinus grafting. Clin Oral Impl Res 2001; 12: 488494.

11. Kanno, T., Takabashi, T., Tsujisawa, T., Ariyoshi, W., Nishihara, T.: Platelet-Rich Plasma enhances human osteoblast-like cell proliferation and differentation. J Oral Maxillofac Surg. 2005; 63: 362-369.

12. Lang, E.E., Curran, A.J., Patil, N., Walsh, R.M., Rawluk, D., Walsh, M.A.: Intracranial complications of acute frontal sinusitis. Clin Otolaryngol. 2001; 26: 452-457.

13. Lee, W.C.: Recurrent frontal sunusitis complicating orbital decompression in Graves' disease. J Laryngol Otol. 1996; 110: 670- 672.

14. Maniglia, A.J., Goodwin, W.J., Arnold, J.E., Ganz, E.: Intracranial abscesses secondary to nasal sinus, and orbital infections in adult and children. Arch Otolaryngol Head Neck Surg. 1989; 115: 1424-1429.

15. Mazock, J., Schow, S., Triplett, R.: Proximal tibia bone harvest: Review and technique, complications, and use in Maxillofacial Surgery. Int J Oral Maxillofac Surg. 2004; 19: 586-593.

16. Mendians, A.E., Marks, S.C.: Outcome of frontal sinus obliteration. Laryngoscope. 1999; 109: 1495-1498.

17. Parhiscar, A., Har-El, G.: Frontal sinus obliteration with the pericraneal flap. Otolaryngol Head Neck Surg. 2001; 124: 304-307.

18. Peltola, M., Suonpa, A.J., Aitasalo, K., Varpula, M., Yli-Urpo, A., Happonen, R-P.: Obliteration of the frontal sinus cavity with bioactive glass. Head Neck. 1998; 20: 315-319.

19. Petruzzelli, G.J., StanKiewicz, J.A.: Frontal sinus obliteration with hydroxyapatite cement. Laryngoscope. 2002; 112: 32-36.

20. Rinehart, G.C., Jackson, I.T., Potparic, Z., Tan, R.G., Chambers, P.A.: Management of locally agressive sinus disease using craniofacial exposure and the galeal frontalis fascia-muscle flap. Plast Reconstr Surg. 1993; 92: 12191225.

21. Rohrich, R.J., Mickel, T..J.: Frontal sinus obliteration: 
search of the ideal autogenous material. Plast Reconstr. Surg. 1995; 95 : 580-585.

22. Sailer, H.F., Gritz, K.W., Kalavrezos, N.D.: Frontal sinus fractures: principles of treatment and long term results after sinus obliteration with the use of Lyophilized cartilage. J Cranio-Maxillofac Surg. 1998; 36: 235-342.

23. Sánchez, A., Sheridan, P., Kupp, L.: Is platelet-rich plasma the perfect enhacement factor? A current review. Int J Oral Maxillofac Surg. 2003; 18: 93- 103.

24. Singh, B., Dellen Van, J., Ranjettan, S., Maharaj, T.J.: Sinogenic intracranial complications. J Laryngol Otol. 1995; 109: 945.

25. Weber, R., Draf, W., Keerl, R., Kahle, G., Schinzl, S., Tomann, S., Lawson, W.: Osteoplastic frontal sinus surgery

Comentario al trabajo Obliteración del seno frontal mediante injerto de tibia y plasma rico en plaquetas para el tratamiento de la osteomielitis crónica de M AcostaFeria y cols

El presente trabajo describe el empleo de un injerto autólogo de hueso esponjoso tibial asociado a plasma rico en plaquetas, en la reparación quirúrgica del seno frontal. Este tratamiento se empleó en un paciente con una osteomielitis crónica como complicación de una sinusitis secundaria al tratamiento quirúrgico y quimioterápico de un plasmocitoma del seno frontal. Los autores presentan una técnica novedosa (en cuanto al material empleado) para la obliteración del seno frontal. El uso de plasma rico en plaquetas, de acuerdo a unos estándares de calidad, parece favorecer tanto la calidad de la reparación de las áreas receptoras del injerto como la velocidad de la reparación ${ }^{1}$. Cabe preguntarse sin embargo, si debe generalizarse with fat obliteration: technique and long-term results using magnetic resonance imaging in 82 operations. Laryngoscope. 2000; 110: 1037-1044.

Acosta-Feria, M.; Infante-Cossío, P.; Hernández-Guisado, J.M.; Torres-Carranza, E.; García-Perla, A.; BelmonteCaro, R.; Gutiérrez-Pérez. J.L.: Obliteración del seno frontal mediante injerto de tibia y plasma rico en plaquetas para el tratamiento de la osteomielitis crónica. Neurocirugía 2006; 17: 351-356.

Correspondencia postal: Dr. Manuel Acosta Feria. Servicio de Cirugía Maxilofacial. Hospitales Universitarios Virgen del Rocío. Av. Manuel Siurot sin. 41013 Sevilla.

el uso de estas sustancias ricas en factores de crecimiento o si su uso se debe restringir a situaciones clínicas peculiares en que la reparación mediante técnicas convencionales presente dificultades.

\section{Bibliografía}

1. Marx, R.E.: Platelet-Rich plasma: Evidence to support its use. J Oral Maxillofac Surg 2004; 62: 489-496.

A. Pérez Núñez

R. D. Lobato

Madrid 